\title{
Mitochondrial fusion fuels T cell memory
}

\author{
Cell Research (2016) 26:969-970. doi:10.1038/cr.2016.94; published online 12 August 2016
}

\section{Differences in mitochondrial struc- tures determine the metabolic land- scape of effector and memory $T$ cell populations in vivo.}

Proliferating lymphocytes and cancer cells use the 'Warburg effect' in which cells switch from more energy-efficient oxidative phosphorylation (i.e., the mitochondrial break-up of nutrients) to aerobic glycolysis (i.e., the cytoplasmic oxidation of glucose into pyruvate) to generate building blocks for growth. Immunological memory following the proliferative expansion of antigenspecific clones, in contrast, requires a switch back to mitochondrial oxidative phosphorylation to gain the capacity for long-term survival. However, mechanisms underlying this intrinsic metabolic plasticity had remained elusive. Pearce and colleagues show that fission and fusion forces regulate mitochondrial function in T lymphocytes, thereby linking mitochondrial reprogramming and determination of fate in lymphocytes [1]. These new findings extend previous work on multiple specialized roles of mitochondria in different stages of the immune response [2].

Buck et al. [1] show that effector T cells with fissed mitochondria display disorganized cristae, while memory $\mathrm{T}$ cell populations have fused mitochondria and better-organized cristae. Importantly, enforcing mitochondrial fusion in effector $\mathrm{T}$ cells (by genetic or pharmacologic manipulation) led to metabolic reprogramming, with enhanced oxidative metabolism, increased T cell survival and improved anti-cancer activity in vivo. These data allocate metabolic dynamics downstream of $\mathrm{T}$ cell activation (needed to establish an effector phenotype), but upstream of metabolic commitment. That is, effector $\mathrm{T}$ cell populations require mitochondrial re-arrangements (fission) to engage in aerobic glycolysis. Thus, inhibition of fission enforces memory-like $\mathrm{T}$ cell properties even in the presence of an upstream activation trigger. This approach may have important consequences to improve the generation of immunological memory.

Nevertheless, enforcing immunological memory by means of the newlydiscovered fusion program may not be always ideal. Primary immunizations, i.e., influenza vaccination, may be reduced by excessive oxidative metabolism while strongly dependent on effector T cell priming of B cells. Taken together, these findings enhance our understanding of how metabolic switches and $\mathrm{T}$ cell activities are linked, and illuminate mitochondrial reprogramming as a strategy to modulate T cell function.

There have been a number of other roles for mitochondrial dynamics in $\mathrm{T}$ cell activation and most of this information fits well with the new paradigm introduced by Buck et al. [1]. It is well established that mitochondria play an important role in $\mathrm{Ca}^{2+}$ homeostasis. The electromotive force of the energized inner membrane can move large amount of $\mathrm{Ca}^{2+}$ into the matrix, but only when cytoplasmic $\mathrm{Ca}^{2+}$ reaches a high local concentration [2]. An important function of this $\mathrm{Ca}^{2+}$ buffering is to keep plasma membrane Orai channels open longer by avoiding $\mathrm{Ca}^{2+}$-dependent closure [2]. The mitofusin-2-dependent adhesion of the endoplasmic reticulum to mitochondria, which is also part of the mitochondrial fusion apparatus, also contributes to $\mathrm{Ca}^{2+}$ flux, by positioning mitochondria near inositol-3-phosphate receptors [3]. Thus, completely de-energizing mitochondria tends to shut down or lead to oscillations in the sustained cytoplasmic $\mathrm{Ca}^{2+}$ in response to activation [2]. The phenotype of stomatin-like protein 2 (SLP-2) knockout mice also supports an important role of mitochondrial integrity for T cell activation [4]. In addition to $\mathrm{Ca}^{2+}$ buffering, mitochondria have been implicated in polarized release of extracellular ATP [5], which provides another way for mitochondria to contribute to $\mathrm{Ca}^{2+}$ flux.

The impact of manipulating mitochondrial fission and fusion on $\mathrm{T}$ cell migration and the immunological synapse has also been investigated. $\mathrm{T}$ cell migration is related to the immunological synapse in that they share a similar actinomyosin machinery to drive directed motility and the T cell receptor/ adhesion bull's eye [6]. T cell migration and mitochondrial polarization are promoted by mitochondrial fission [7]. A similar relationship has been observed in the immunological synapse, where dynamin-related protein-1 (Drp1) is concentrated in the synapse and mediates fission [8]. These studies struck a similar cord by suggesting that an important local function of polarized mitochondria is to provide ATP for the myosin motors. Some aspects of immunological synapse formation are actually involved in turning off TCR signaling, such that suppressing Drp1-mediated fission actually enhanced $T$ cell activation [8]. A caveat of these studies is that they made extensive use of the Jurkat $\mathrm{T}$ cell line, and these issues will need to be further explored with primary T cells. 
Figure 1 shows the accumulation of mitochondria near the secretory domain of the immunological synapse formed by non-transformed mouse effector $\mathrm{T}$ cells as revealed by electron tomography [9].

Asymmetric partition of proteins has been recently shown to shape the distinct metabolic landscape of effector and memory $\mathrm{T}$ cell populations during antigen presentation [10]. Mitochondria can also be inherited during cell division [11]. It remains to be determined whether any distribution of differently structured mitochondria with tighter or looser cristae, may also have an impact on $\mathrm{T}$ cell differentiation or whether differences in mitochondrial structure are actively acquired in response to the recently described asymmetric programs.

Several questions still remain. Above all, how mitochondrial fusion, oxidative metabolism and immunological memory are exactly linked? Pearce and colleagues show that enforcing fusion can enhance oxidative phosphorylation with consequent activation of fatty acid oxidation in T cells (FAO; the mitochondrial breakdown of lipids thought to fuel long-term memory survival). However, $\mathrm{T}$ cells with a genetic defect in fusion (missing the key fusion protein OPA1) could still activate FAO, suggesting the involvement of additional mechanisms. Second, how the predominant role of fusion in memory $\mathrm{T}$ cell generation and fatty acid metabolism can be re-conciliated with the metabolism of highly differentiated (memory) $\mathrm{T}$ cells (also known as senescent $\mathrm{T}$ cells) that spontaneously activate AMPK (a key activator of FAO) but that present poor mitochondrial metabolism [12]? Third, how changes in fusion/fission (and hence in $\mathrm{T}$ cell metabolism) may

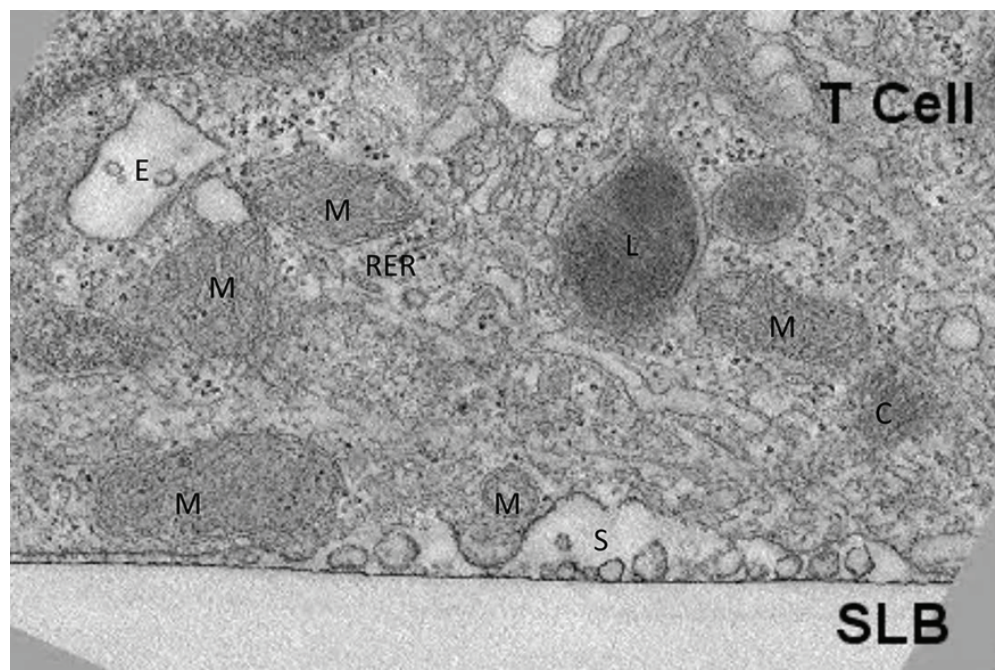

Figure 1 Accumulation of mitochondria at the immunological synapse. Electron tomography of immunological synapse formed on suppored lipid bilayers (SLB) containing ICAM-1 and agonist MHC peptide complexes [9]. M, mitochondria; C, centriole; $E$, endosome; L, lysosome; $S$, secretory domain; RER, rough endoplasmic reticulum.

help to differentiate responses of naïve, memory and senescent $T$ cells? While the loss of Drp1 from the Jurkat T cells enhanced IL-2 production, the fusion proteins have not been similarly manipulated and little work has been done on these aspects in primary $\mathrm{T}$ cells. In addition, AMPK activation also inhibits TCR sensitivity. Thus, understanding how metabolic processes feed into the immunological synapse may unveil unrecognized strategies for intervention during aging, chronic viral infections and cancer.

\footnotetext{
Alessio Lanna ${ }^{1}$, Michael L Dustin ${ }^{1}$

${ }^{1}$ Kennedy Institute of Rheumatology, NDORMS, University of Oxford, Oxford OX3 7FY, UK Correspondence: Michael L Dustin

E-mail: michael.dustin@kennedy.ox.ac.uk
}

\section{References}

1 Buck MD, O'Sullivan D, Klein Geltink RI, et al. Cell 2016; 166:63-76.

2 Hoth M, Fanger CM, Lewis RS. J Cell Biol 1997; 137:633-648.

3 de Brito OM, Scorrano L. Nature 2008; 456:605-610.

4 Christie DA, Kirchhof MG, Vardhana S, et al. PLoS One 2012; 7:e37144.

5 Ledderose C, Bao Y, Lidicky M, et al. J Biol Chem 2014; 289:25936-25945.

6 Dustin ML. Curr Opin Cell Biol 2007; 19:529-533.

7 Campello S, Lacalle RA, Bettella M, et al. J Exp Med 2006; 203:2879-2886.

8 Baixauli F, Martin-Cofreces NB, Morlino $\mathrm{G}$, et al. EMBO J 2011; 30:1238-1250.

9 Choudhuri K, Llodra J, Roth EW, et al. $\mathrm{Na}$ ture 2014; 507:118-123.

10 Verbist KC, Guy CS, Milasta S, et al. Nature 2016; 532:389-393.

11 Katajisto P, Dohla J, Chaffer CL, et al. Science 2015; 348:340-343.

12 Lanna A, Henson SM, Escors D, et al. Nat Immunol 2014; 15:965-972. 